\title{
Laboratory assessments of therapeutic platelet inhibition in endovascular neurosurgery: comparing results of the VerifyNow P2Y12 assay to thromboelastography with platelet mapping
}

\author{
Brian M. Corliss, MD, ${ }^{1}$ Adam J. Polifka, MD, ${ }^{1}$ Neil S. Harris, MD, MBChB,${ }^{2}$ Brian L. Hoh, MD, ${ }^{1}$ and \\ W. Christopher Fox, MD'
}

Departments of ${ }^{1}$ Neurological Surgery and ${ }^{2}$ Pathology, University of Florida, Gainesville, Florida

\begin{abstract}
OBJECTIVE Inhibition of platelet aggregation is vital to preventing thromboembolic complications related to stent placement in endovascular neurosurgery, but excessive inhibition potentiates hemorrhagic complications. Recent evidence suggests an ideal inhibition range of 70-150 P2Y12 response units (PRU) as measured on the VerifyNow assay, which relies on photometric measurements of platelet aggregation. Thromboelastography (TEG) with platelet mapping (PM) is an alternative assay that directly measures clot formation and mechanical strength. This study compares the results of PRU to TEG-PM.
\end{abstract}

METHODS Patients with simultaneous or near-simultaneous PRU and TEG-PM results who underwent cervical carotid artery stenting, intracranial stent-assisted aneurysm coiling, or flow diversion at the authors' institution between August 2015 and November 2016 were identified. PRU results were compared with the TEG maximal amplitude (MA) attributable to adenosine diphosphate (ADP) activity (MA-ADP) as measured by TEG-PM. Platelet inhibition was considered therapeutic for MA-ADP values $<50 \mathrm{~mm}$ or PRU $<194$. The Pearson correlation coefficient was calculated, and the sensitivity and specificity of PRU were calculated assuming that the results of TEG-PM reflected the true degree of platelet inhibition.

RESULTS Twenty-three patients were identified with a total of 37 matched sets of TEG-PM and PRU. Three of these pairs were excluded due to anemia outside of the PRU manufacturer's recommended range. The Pearson correlation coefficient for these values was $0.50(p=0.0026)$. The prevalence of clopidogrel nonresponders determined by TEG-PM (9\%) matched reported rates (5\%-12\%); PRU demonstrated much higher prevalence (39\%). For detecting a therapeutic level of platelet inhibition, PRU demonstrated a sensitivity of 0.59 , specificity of 0.50 , positive predictive value of 0.95 , and negative predictive value of 0.07 . Ideal inhibition was concordant in only $25 \%$ of observations in which at least one of the results was ideal.

CONCLUSIONS Agreement between TEG-PM and PRU regarding the degree of platelet inhibition is poor. PRU likely overestimates clopidogrel resistance, as $93 \%$ of patients with PRU > 194 demonstrate a therapeutic level of platelet inhibition on TEG.

https://thejns.org/doi/abs/10.3171/2017.6.JNS17535

KEY WORDS therapeutic platelet inhibition; dual antiplatelet therapy; endovascular surgery; clopidogrel; nonresponder; resistance; vascular disorders

$\mathrm{T}$ HE armamentarium of devices available to the endovascular neurosurgeon has blossomed and evolved significantly over time, and now includes a wide range of endoluminal stents requiring dual antiplatelet therapy (DAPT) for platelet thrombus prophylaxis prior to deployment. Over time, first-line DAPT has continued to consist primarily of the irreversible cyclooxygenase (COX) antagonist aspirin and the irreversible P2Y12 adenosine diphosphate (ADP) receptor antagonist clopidogrel, despite the fact that $5 \%-12 \%$, 11 of patients are clopidogrel

ABBREVIATIONS $\mathrm{AA}=$ arachidonic acid $\mathrm{ADP}=$ adenosine diphosphate $;$ COX = cyclooxygenase; $\mathrm{DAPT}=$ dual antiplatelet therapy; $\mathrm{MA}=$ maximal amplitude; $\mathrm{PED}=\mathrm{Pipe}-$ line embolization device; $P F T=$ platelet-function testing; $P M=$ platelet mapping; $P R U=P 2 Y 12$ response units; TEG = thromboelastography .

SUBMITTED February 28, 2017. ACCEPTED June 5, 2017.

INCLUDE WHEN CITING Published online December 22, 2017; DOI: 10.3171/2017.6.JNS17535. 
nonresponders, and many more demonstrate suboptimal platelet inhibition in response to the drug. To combat this, many endovascular surgeons use some form of laboratory assay of P2Y12 receptor activity. Until now, the VerifyNow P2Y12 assay (PRU, Accumetrics) has been the best studied and most widely used of these tests, but our anecdotal experience with this test for this application has shown it to be somewhat unreliable. Additionally, the PRU is of unclear applicability outside of this context, as it has not been studied in other neurosurgical patients on antiplatelet agents, such as patients undergoing emergency surgery or patients presenting with intracranial hemorrhage. PRU also cannot differentiate aspirin-induced platelet inhibition in patients administered clopidogrel.

As an alternative, thromboelastography (TEG) with platelet mapping (PM) has been used extensively in cardiovascular and trauma surgery to manage anticoagulation and antiplatelet therapy, as it can be used to detect $\mathrm{P} 2 \mathrm{Y} 12$ receptor inhibition in addition to platelet inhibition through the COX-arachidonic acid (AA) pathway and enzymatic coagulopathy. In TEG, nascent thrombus integrity is measured mechanically and in real time as blood clots. A mechanical probe is deflected by the thrombus during the process of coagulation, generating a characteristic curve; platelet aggregation contributes to a measured feature of this curve called the maximal amplitude (MA), measured in millimeters. In TEG-PM, the test is run 4 times in the presence of different reagents to selectively inhibit or promote different platelet-activating chemical pathways. In conducting this test in this manner, the activities of the P2Y12 receptor and the COX-AA pathway can be independently assessed, and the MA attributable to ADP (MA-ADP) or AA (MA-AA), and relative percentage of ADP-receptor or AA-receptor inhibition can be determined.

Unfortunately, there is a dearth of experience with this test in neurosurgical patients, and no literature exists comparing the results of TEG-PM to PRU in these patients. When TEG-PM has been used in the literature, it has frequently been in the form of percentage of ADP inhibition rather than MA-ADP, and little is known about whether a one-to-one relationship exists between these 2 values, as percentage of ADP inhibition is a mathematically derived parameter that is dependent on baseline coagulation characteristics. In the present study, we compare the results of the PRU to TEG-PM in a cohort of neurosurgical patients undergoing cerebrovascular interventions requiring DAPT.

\section{Methods \\ Study Population}

After obtaining IRB approval, the electronic medical record was used to retrospectively identify patients who had undergone an endovascular neurosurgical procedure requiring DAPT. These cases included cervical carotid artery stenting, Pipeline embolization device (PED; Covidien/ev3) insertion, stent-assisted coiling, and venous sinus stenting procedures. The medical record was then used to identify and exclude patients who did not have simultaneously or near-simultaneously recorded PRU and
TEG-PM results dating back to September 2015, when TEG-PM began to be used at our institution. TEG-PM and PRU were not both routinely collected simultaneously by all attending surgeons until November 2016. Prior to this date, however, they were routinely collected on all patients by 2 of the 3 attending surgeons (A.J.P. and W.C.F.). All patients whose data were used for this study had both PRU and TEG-PM laboratory results collected as a routine part of their preoperative workup. Patients were not enrolled if one laboratory test was collected to supplement the other after an abnormality was discovered. All patients were routinely initiated on DAPT 7-10 days prior to their scheduled procedure. DAPT regimens were determined at the discretion of the attending surgeon, but generally consisted of either 81 or $325 \mathrm{mg}$ of aspirin and $75 \mathrm{mg}$ of clopidogrel daily.

\section{Data Collection}

Once patients were identified, simultaneously collected PRU and TEG-PM results were tabulated. The most recent complete blood count results obtained prior to the PRU result were also recorded, and PRU/TEG-PM paired results were excluded if the hematocrit level was less than $30 \%$ or the platelet count was less than $115,000 / \mathrm{mm}^{3}$, as these results are outside of the PRU test manufacturer's suggested reference values. PRU is known to be inaccurate outside of these hematological reference ranges. ${ }^{6} \mathrm{MA}$ ADP and percentage of ADP inhibition obtained from TEG-PM were plotted relative to one another to assess the relationship between these terms. MA-ADP was then plotted relative to PRU, and this relationship was then used to calculate binary performance statistics using the lower limit of the manufacturers' reference ranges-194 PRU or $50 \mathrm{~mm}$ - for PRU and TEG-PM, respectively, with the assumption that TEG-PM reflected the true degree of ADP receptor inhibition. The method of least squares was used to create linear trend lines for prognostication, and Pearson correlation coefficients were calculated. Fisher's R-to$\mathrm{Z}$ transform was then used to determine the significance of the correlation and to compare Pearson coefficients. A 2-tailed Fisher's exact test was used to compare binary variable results.

\section{Results}

Twenty-three patients were retrospectively identified with 37 pairs of PRU and TEG-PM results. Three of the paired results $(8 \%)$ were discarded due to anemia. All patients had at least 1 pair of results obtained in the absence of significant anemia or thrombocytopenia. We then used the manufacturers' suggested reference values for the lower limit of normal (194 PRU or $50 \mathrm{~mm}$ ) as the threshold to detect response to clopidogrel. In this analysis, we found $9(39 \%)$ of 23 patients were clopidogrel nonresponders as measured by PRU, compared with $2(9 \%)$ of 23 as measured by TEG-PM. The difference between these results was significant $(p=0.035)$. The incidence of clopidogrel nonresponders as determined by TEG-PM was within the established literature estimate of 5\%-12\%.

Given that both percentage of ADP inhibition and MAADP have been used previously in studies of TEG-PM, 


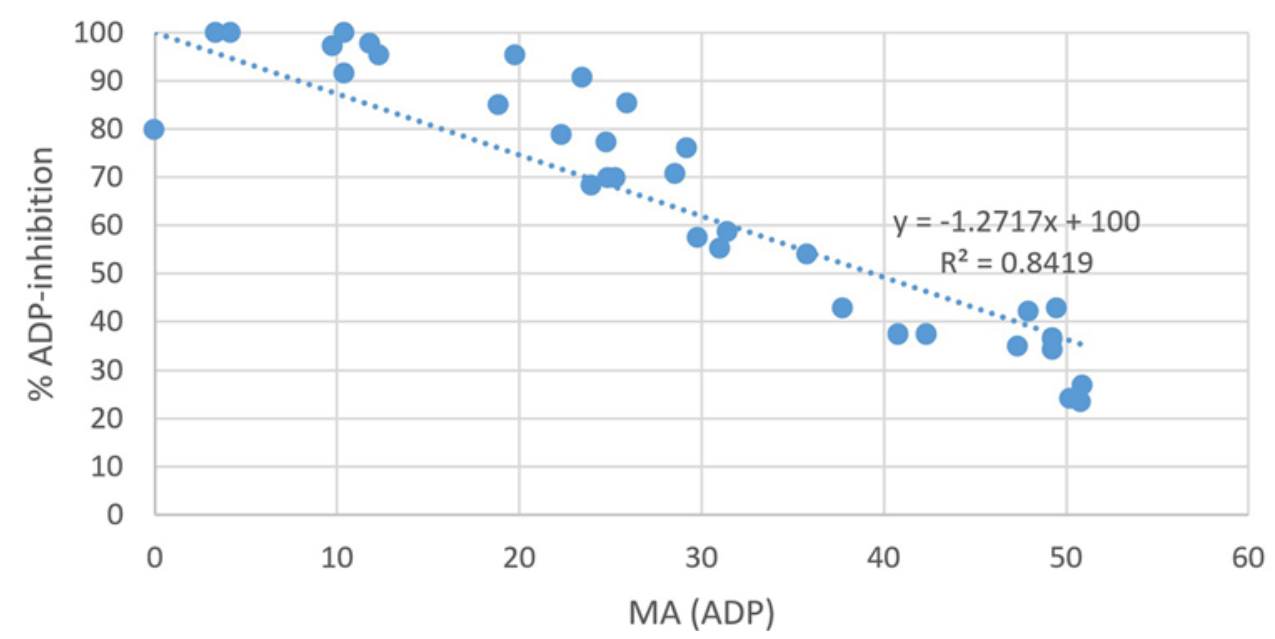

FIG. 1. Linear relationship between the percentage of ADP inhibition and MA-ADP. Note that the y-intercept has been set to $100 \%$ inhibition for the purpose of trend line fitting. The equation $(y=-1.2717 x+100)$ represents the best-fit line, used to calculate the predicted MA-ADP from a given percentage inhibition and vice versa. Figure is available in color online only.

and that percentage of ADP inhibition is a mathematically derived value that is dependent on factors aside from the MA-ADP alone, we next sought to compare the results of these tests to determine if using them interchangeably was valid. We plotted the results of these tests (Fig. 1) and fit a linear trend line, which demonstrated a high degree of linear correlation.

We next compared the results of simultaneously ac- quired MA-ADP, percentage of ADP inhibition, and PRU graphically (Fig. 2) and again fit linear trend lines to assess degree of correlation. Although both MA-ADP and percentage of ADP inhibition demonstrated linear correlation with PRU that was highly significant $(\mathrm{p}<0.01)$, the degree of correlation was weak in both cases. There was no significant difference in the correlation coefficients $(\mathrm{p}$ $=0.83$ ), indicating that MA-ADP and percentage of ADP
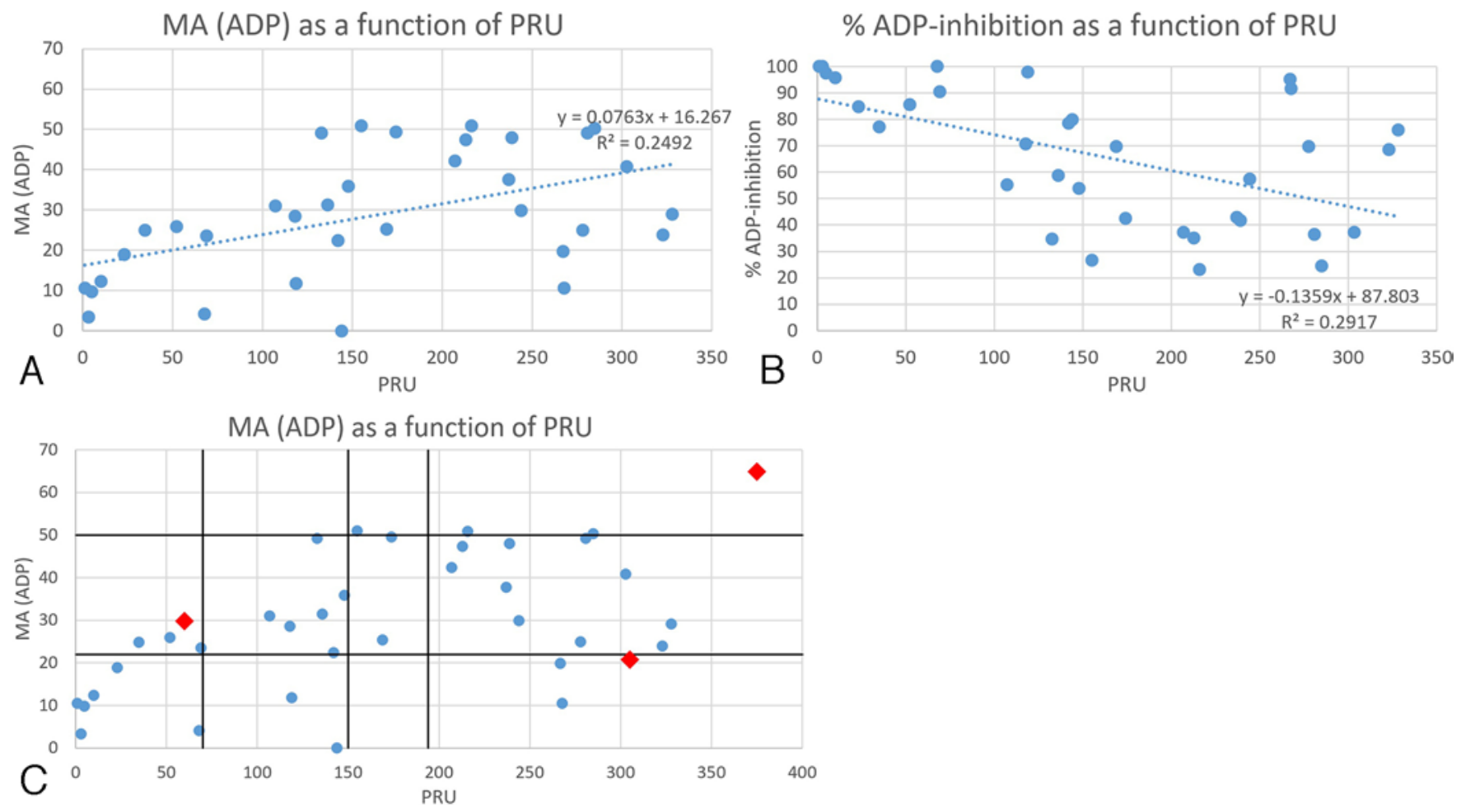

FIG. 2. $A$ and B: Scatterplots demonstrating the relationship between MA-ADP and PRU (A) and percentage of ADP inhibition and PRU (B). No significant difference was found between the degrees of correlation. C: Scatterplot with superimposed cutoffs indicating the therapeutic ranges of each test. Red diamonds indicate values excluded due to anemia outside of the PRU manufacturer's reference range. Figure is available in color online only. 
inhibition were similarly well correlated with the PRU result.

Previous nonrandomized studies in cardiac surgery ${ }^{12}$ and endovascular neurosurgery ${ }^{13,14}$ have demonstrated an MA-ADP of $50 \mathrm{~mm}$ as an upper safe threshold above which thromboembolic complications are more likely. No studies of TEG have demonstrated a safe lower threshold, but a lower limit of 70 PRU has been demonstrated as a safe threshold below which hemorrhage is more likely in patients undergoing PED placement in one nonrandomized study. ${ }^{3}$ Using the trend lines fitted in Fig. 2, we then estimated the percentage of ADP inhibition and MA-ADP correlating with 70 PRU. This yielded results of $78 \%$ ADP inhibition and $22 \mathrm{~mm}$, respectively. Using the trend line in Fig. 1, we also calculated that $50 \mathrm{~mm}$ correlated approximately with 36\% ADP inhibition. These results suggest an ideal range of 22-50 mm for MA-ADP and 36\%-78\% ADP inhibition in patients undergoing endovascular procedures requiring DAPT.

We next compared the results of the PRU to MA-ADP to establish performance characteristics of the PRU, assuming the TEG-PM results reflected the true degree of inhibition. With respect to presence of platelet inhibition, PRU demonstrated a sensitivity of 0.59 , specificity of 0.50 , positive predictive value of 0.95 , and negative predictive value of 0.07 . Assuming ideal inhibition by TEG-PM of 22-50 mm MA-ADP and ideal inhibition by PRU of $70-150$, we identified 16 of 74 tests demonstrating ideal inhibition by either measure. Of these 16 tests, 4 (25\%) demonstrated agreement that inhibition was ideal on both TEG-PM and PRU.

\section{Discussion}

Endoluminal devices-typically bare-metal stentshave become ubiquitous in endovascular neurosurgery. Although these stents have their roots in similar-appearing devices originally introduced in interventional cardiology, an ever-increasing number of novel devices with specific neurovascular indications have arrived on the market. Devices in use range from carotid artery stents for treatment of cervical carotid artery stenoocclusive disease (an area in which interventional neuroradiology, endovascular neurosurgery, interventional cardiology, and peripheral endovascular surgery overlap), to low metal surface area buttressing stents for intracranial use as adjuncts to standard microcoil embolization techniques, to flow diverters such as the PED, to the new frontier of combination buttress/flow-diverting devices like the PulseRider (Codman/ Pulsar Vascular), which is still under clinical investigation and not currently available in the US. ${ }^{2}$ These devices have the unfortunate downside of requiring DAPT prior to use.

Given the high prevalence of clopidogrel nonresponders and hyporesponders, and the potentially devastating effects of stent occlusion, in-stent stenosis, or distal thromboembolism in endovascular neurosurgery, many centers routinely use laboratory tests to confirm adequate platelet inhibition in patients undergoing these procedures. In this field, the most widely used laboratory assessment to date has been the VerifyNow P2Y12 receptor assay. ${ }^{3-5,10}$ This test utilizes a small-volume spectrophotometric platelet aggregometer in a point-of-care device. Encouragingly, a recent study ${ }^{3}$ involving 231 patients harboring 248 predominantly unruptured aneurysms treated with PEDs demonstrated that PRU values in the 60-240 range (and ideally in the 70-150 PRU range) prior to embolization resulted in fewer thromboembolic and hemorrhagic complications. However, a meta-analysis ${ }^{10}$ of published results prior to that publication did not find any reduction in ischemic or hemorrhagic complications following PED placement for patients undergoing preoperative plateletfunction testing (PFT) using PRU. Another study ${ }^{4}$ did not detect any increase in thromboembolic complications for PRU in the 200-240 range compared with 60-200, although this study was likely underpowered to detect such a difference. No randomized controlled trials exist demonstrating increased safety or efficacy of treatment in patients whose response to clopidogrel has been monitored by means of any laboratory test. Despite the widespread use of PFT, it is not considered necessary to perform this assessment. Some surgeons and interventionalists do not routinely monitor platelet inhibition. Given the wide variability of the results of the PRU, one can understand why this approach would be adopted. Anecdotally, we have seen hemorrhagic complications resulting from medication manipulations performed in response to subtherapeutic platelet-function assessments, further demonstrating the need for accurate and precise assessment of platelet function that is necessary if these technologies are going to become the standard of care and increase patient safety.

TEG-PM is widely used in cardiac and general surgery, and has been demonstrated in these fields to accurately predict bleeding and thromboembolic complications. In a recent pilot study ${ }^{12}$ of TEG-PM for anticoagulation and antiplatelet management in patients receiving mechanical circulatory support devices (e.g., left ventricular assist devices), MA-ADP and MA-AA were thought to be ideal if below $50 \mathrm{~mm}$. In another study, ${ }_{1}^{1}$ patients undergoing coronary artery bypass grafting procedures were randomized to undergo preoperative assessment of platelet function using aggregometry (the gold-standard nonclinical laboratory assessment of platelet function), TEG-PM, or none. Patients undergoing either of the PFTs had less intraoperative blood loss, were less likely to require red cell or platelet transfusions, were transfused fewer units of blood products when transfusion was required, and were less like to require resternotomy. There was also a financial savings associated with preoperative utilization of PFTs if the patient was taking an ADP receptor antagonist prior to surgery. A case-control study ${ }^{7}$ of noncardiac general surgery patients undergoing operations within 7 days of taking clopidogrel demonstrated that preoperative percentage of ADP inhibition $>30 \%$ was correlated with increased transfusion requirements, increased likelihood of requiring reoperation, and a trend toward increased risk of death.

Reports in endovascular neurosurgery in Chinese patients undergoing stent placement for intra- or extracranial vascular stenosis ${ }^{13}$ and stent-assisted aneurysm coiling ${ }^{14}$ have demonstrated a correlation between TEG-PM values and the risk of thromboembolic complications. Specifically, although no difference was noted between pa- 
tients with thromboembolic complications in terms of the aspirin-induced platelet inhibition, in both patient cohorts an MA-ADP $>50 \mathrm{~mm}$ correlated with a marked increase in thromboembolic complications ( $20 \%$ vs $6 \%$ ). The only report ${ }^{8}$ of TEG-PM being used in the US for neuroendovascular patients used arbitrary cutoffs of $90 \%$ ADP inhibition or 10\% ADP inhibition to define hyperinhibition or inadequate inhibition, respectively, and tailor DAPT regimens. There were no clinically apparent complications in 31 patients undergoing PED insertion, indicating that TEG-PM may be used safely to guide decisions on platelet therapy.

None of the presently available literature has directly compared TEG-PM to PRU. In our study, we have compared the results of these 2 tests directly to demonstrate that there is very poor inter-test reliability. TEG-PM appears to correlate well with established estimates of the prevalence of clopidogrel nonresponders, and we therefore used it to represent the true measure of therapeutic platelet inhibition in our cohort. By doing so, we were able to estimate that PRU has a sensitivity of 0.59 , specificity of 0.50 , positive predictive value of 0.95 , and negative predictive value of 0.07 . These values indicate that a therapeutic PRU value (i.e., < 194) is highly likely to indicate true platelet inhibition in these patients. However, a subtherapeutic value (i.e., > 194) is more likely to be a false negative than to truly indicate dangerously uninhibited platelet function. Of course, the assumption that TEG-PM represents the true platelet function is subject to question, as this has never been proven superior to PRU in head-to-head testing. This should be investigated in a rigorous manner in the future. However, in our cohort, TEG-PM results correlated to a much greater degree with previously published data than did PRU. In addition, in our clinical experience, we have found TEG-PM to be much more consistent than PRU and have begun using this test to help guide clinical decision making.

Our trend line analyses have demonstrated a strong correlation between MA-ADP and percentage of ADP inhibition, indicating that either of these tests can be used going forward for assessment of platelet inhibition. We favor the MA-ADP, as this has been more rigorously tested with respect to thromboembolic complications in the neuroendovascular literature from China, ${ }^{13,14}$ and because the percentage of ADP inhibition is a mathematically derived value that depends on a patient's baseline coagulation profile. In principle, there could be a patient whose baseline platelet function is on the lower end of normal who might have a therapeutic MA-ADP with a relatively low percentage inhibition. Nonetheless, based on the present study and previously published results, we recommend an ideal preoperative therapeutic range of $20-50 \mathrm{~mm}$ or $35 \%-80 \%$ inhibition for MA-ADP and percentage of ADP inhibition, respectively, for endovascular neurosurgery patients requiring DAPT. We do feel, however, that an assessment of complications in a larger sample of patients will be required to confirm the utility of TEG-PM values in endovascular neurosurgery. Furthermore, in our practice, we continue to obtain both PRU and TEG-PM on all patients and interpret their results simultaneously.

Whether these data might be useful outside of endovas- cular neurosurgery remains to be seen. TEG has become widely used for coagulopathy monitoring in patients with coagulopathy in other fields of medicine (e.g., trauma, liver disease/transplant, oncology, etc.). This technology may be applicable to nonvascular neurosurgical patients as well. Knowledge of the TEG-PM could add significantly to the management of patients with spontaneous intracerebral hemorrhage, extraaxial hematomas in older patients receiving antiplatelet agents, and in many other neurosurgical patients who are prescribed these medications prior to surgery. If the utility of this test is shown to be useful in one subset of neurosurgical patients, such as endovascular patients on DAPT, the broader applicability of TEG-PM may established by similar means going forward.

The present study is limited by the relatively small patient size, and the lack of gold-standard testing. Although we have demonstrated that TEG-PM appears to result in data that more closely reflect what has previously been determined regarding clopidogrel resistance, to determine which test should be considered the gold standard would require a clinical assessment of complications. With such a limited sample size, it would be futile to attempt to discern a significant difference with respect to thrombotic or hemorrhagic complications presently. However, it is now standard of care at our institution to collect both PRU and TEG-PM data for all patients undergoing endovascular procedures requiring DAPT, so accrual of patients for future evaluation should be much more rapid than it has been up to now. Furthermore, because all patients will have these data, this should eliminate any potential for selection bias introduced by retrospectively reviewing data obtained outside of a systematic protocol. These data may also be valuable for determining whether aspirin-induced platelet inhibition is a significant factor when attempting to prevent complications in this group of patients, a factor that has not previously been studied, and that can only be ascertained through the use of TEG-PM.

One other limitation is the possibility for selection bias given that PRU and TEG-PM were not routinely collected on all endovascular neurosurgical patients on DAPT during the study period. However, all patients enrolled in the study had both laboratory results collected as a matter of routine practice. Two of the 3 authors contributing patients (W.C.F. and A.J.P.) routinely collected both laboratory results on all of their patients for the entirety of the study period, and all but 3 of the enrolled patients were treated by these 2 surgeons. The other 3 patients were enrolled by the third surgeon (B.L.H.) after the introduction of the policy of routinely collecting both laboratory results. Hence, although the subjects enrolled are more likely to be patients of W.C.F. or A.J.P. than of B.L.H., there was not any bias in the selection of which patients were tested with both PRU and TEG-PM, and, therefore, which were eligible for enrollment.

\section{Conclusions}

Agreement between TEG-PM and PRU regarding degree of platelet inhibition is poor. PRU likely overestimates clopidogrel resistance, as 93\% of patients with PRU $>194$ demonstrate a therapeutic level of platelet inhibition 
on TEG-PM. Our data suggest a possible therapeutic reference range of $22-50 \mathrm{~mm}$ MA-ADP or $35 \%-80 \%$ ADP inhibition, although further study is needed to corroborate these ranges with clinically observed ischemic and thromboembolic complications.

\section{References}

1. Agarwal S, Johnson RI, Shaw M: Preoperative point-of-care platelet function testing in cardiac surgery. J Cardiothorac Vasc Anesth 29:333-341, 2015

2. Corliss BM, Hoh BL: Editorial. Initial experience with PulseRider treatment for wide-necked bifurcation aneurysms. J Neurosurg 127:59-60, 2017

3. Daou B, Starke RM, Chalouhi N, Barros G, Tjoumakaris S, Rosenwasser $\mathrm{RH}$, et al: $\mathrm{P} 2 \mathrm{Y} 12$ reaction units: effect on hemorrhagic and thromboembolic complications in patients with cerebral aneurysms treated with the Pipeline embolization device. Neurosurgery 78:27-33, 2016

4. Delgado Almadoz JE, Crandall BM, Scholz JM, Fease JL, Anderson RE, Kadkodayan Y, et al: Pre-procedural P2Y12 reaction units value predicts perioperative thrombotic and hemorrhagic complications in patients with cerebral aneurysms treated with the Pipeline Embolization Device. J Neurointerv Surg 5:iii3-iii10, 2013

5. Heller RS, Dandamudi V, Lanfranchi M, Malek AM: Effect of antiplatelet therapy on thromboembolism after flow diversion with the pipeline embolization device. J Neurosurg 119:1603-1610, 2013

6. Kakouros N, Kickler TS, Laws KM, Rade JJ: Hematocrit alters VerifyNow P2Y12 assay results independently of intrinsic platelet reactivity and clopidogrel responsiveness. J Thromb Haemost 11:1814-1822, 2013

7. Kasivisvanathan R, Abbassi-Ghadi N, Kumar S, Mackenzie $\mathrm{H}$, Thompson K, James K, et al: Risk of bleeding and adverse outcomes predicted by thromboelastography platelet mapping in patients taking clopidogrel within 7 days of non-cardiac surgery. Br J Surg 101:1383-1390, 2014

8. McTaggart RA, Choudhri OA, Marcellus ML, Brennan T, Steinberg GK, Dodd RL, et al: Use of thromboelastography to tailor dual-antiplatelet therapy in patients undergoing treatment of intracranial aneurysms with the Pipeline embolization device. J Neurointerv Surg 7:425-430, 2015

9. Müller I, Besta F, Schulz C, Massberg S, Schönig A, Gawaz M: Prevalence of clopidogrel non-responders among patients with stable angina pectoris scheduled for elective coronary stent placement. Thromb Haemost 89:783-787, 2003

10. Skukalek SL, Winkler AM, Kang J, Dion JE, Cawley CM, Webb A, et al: Effect of antiplatelet therapy and platelet func- tion testing on hemorrhagic and thrombotic complications in patients with cerebral aneurysms treated with the pipeline embolization device: a review and meta-analysis. J Neurointerv Surg 8:58-65, 2016

11. Slavik L, Ulehlova J, Krcova V, Hlusi A, Indrakova J, Hutyra $\mathrm{M}$, et al: Detection of clopidogrel resistance using ADP induced aggregometry with specific inhibitor PGE1. Clin Lab 60:1475-1480, 2014

12. Volod O, Lam LD, Lin G, Kam C, Kalyouthapong K, Mac $\mathrm{J}$, et al: Role of thromboelastography platelet mapping and international normalized ratio in defining "normocoagulability" during anticoagulation for mechanical circulatory support devices: a pilot retrospective study. ASAIO J 63:24-31, 2017

13. Wang B, Li XQ, Ma N, Mo D, Gao F, Sun X, et al: Association of thromboelastographic parameters with post-stenting ischemic events. J Neurointerv Surg 9:192-195, 2017

14. Yang H, Li Y, Jiang Y, Lv X: Thromboelastography for monitoring platelet function in unruptured intracranial aneurysm patients undergoing stent placement. Interv Neuroradiol 21:61-68, 2015

\section{Disclosures}

The authors report no conflict of interest concerning the materials or methods used in this study or the findings specified in this paper.

\section{Author Contributions}

Conception and design: Corliss, Polifka, Hoh, Fox. Acquisition of data: Corliss, Hoh, Fox. Analysis and interpretation of data: Corliss, Harris, Fox. Drafting the article: Corliss. Critically revising the article: Corliss, Fox. Reviewed submitted version of manuscript: Corliss, Fox. Approved the final version of the manuscript on behalf of all authors: Corliss. Administrative/technical/material support: Polifka, Harris. Study supervision: Polifka, Hoh, Fox.

\section{Supplemental Information}

Previous Presentations

Portions of this paper were previously presented on February 21, 2017, at the AANS/CNS Joint Cerebrovascular Section Meeting in Houston, Texas.

\section{Correspondence}

Brian M. Corliss, Department of Neurological Surgery, University of Florida, 1600 SW Archer Rd., Box 100265, Gainesville, FL 32610. email: brianmatthew.corliss@neurosurgery.ufl.edu. 